\title{
Model Dependent Controller for Underwater Vehicle
}

\author{
Wesam M. Jasim
}

Department of Information Technology, College of Computer Science and Information Technology, University of Anbar, Ramadi, Iraq

\section{A B S T R A C T}

In this work, a model dependent control design method based feedback scheme was investigated for autonomous underwater vehicle control. The controller was designed with the nonlinear terms - inertia, hydrodynamic damping, and gravitational, and buoyancy - of the vehicle dynamical model consideration. Then, the model independent controller (PD) was also investigated, with no nonlinear terms consideration. The stability analysis of the proposed model dependent feedback controller was obtained based on a Lyapunov function. The simulation results of the proposed controller were compared with that of PD controller. The comparison shows the validation of the proposed controller.

Index Terms: Model Dependent Controller, PD Controller, Underwater Vehicle

\section{INTRODUCTION}

An AUV is a robot able to work in six degrees of freedom with actuators and sensors diving autonomously under the water to perform tasks. The dynamics of the underwater vehicle are nonlinear and acted to disturbances. However, to perform its tasks quickly and accurately, two important problems faced the researchers. They are the problem of identifying the accurate model and designing the suitable control techniques. Therefore, researchers in the robotic control field have been attracted to build their algorithms to solve these problems. In this paper, the problem of building the control algorithm of the underwater vehicle is addressed.

Recently, different control techniques were presented, the $\mathrm{H}_{\infty}$ approach for linear parameter varying polytopic systems was addressed to guarantee the performance of the vehicle [1]. An

\begin{tabular}{|l|l|}
\hline \multicolumn{2}{|c|}{ Access this article online } \\
\begin{tabular}{|l|l|} 
DOI: 10.21928/uhdjst.v1n2y2017.pp25-30 & E-ISSN: 2521-4217 \\
P-ISSN: 2521-4209
\end{tabular} \\
\hline
\end{tabular}

Copyright ( 2017 Jasim. This is an open access article distributed under the Creative Commons Attribution Non-Commercial No Derivatives License 4.0 (CC BY-NC-ND 4.0) optimal control with game theory was presented for position control problem of the underwater vehicle in Patel et al. [2].

Several controllers were discussed in Ferreira et al. [3], some of them were based on Lyapunov control theory, and the others were gathered with linear or nonlinear control theory for underwater vehicle horizontal and vertical motions. A feedback control algorithm was presented in Vervoort [4] to stabilize the underwater vehicle with the linearized model.

A linear quadratic regulator (LQR) algorithm was implemented in Prasad and Swarup [5] for underwater vehicle stabilization combined with Model Predictive control (MPC) for position and velocity control. The simulation results showed a stable response compared with a sliding mode controller performance. While a LQR controller was presented for depth control problem of an underwater vehicle in Joo and $\mathrm{Zu} \mathrm{[6].} \mathrm{The} \mathrm{simulation} \mathrm{results} \mathrm{show} \mathrm{the} \mathrm{success} \mathrm{of} \mathrm{the}$ proposed algorithm.

Authors in Mohd-Mokhtar et al.'s [7] study present a PID controller for underwater vehicle identified model. The simulation results show that the controller performs accurately when the identified model error was 98\% compared with that when the error was $70 \%$.

Corresponding author's e-mail:wmj_r@yahoo.com

Received: 10-03-2017

Accepted: 25-03-2017

Published: 29-08-2017 
Nonlinear control laws were developed in Elnashar [8] for the six degrees of freedom of an underwater vehicle in several motion strategies. The stability of the system was analyzed based on phase plane analysis. An adaptive signal for the unknown forces compensation gathering with a following controller in a limited space for an autonomous vehicle was proposed in Mukheriee et al. [9] without state velocity measurement.

A MPC controller was presented for an AUV low speed tracking control in Steenson et al. [10]. The controller was tested in simulation, and the results were verified by testing the strategy in a tank at zero speed. Authors in Rathore and Kumer [11] study proposed a PID controller for an underwater vehicle steering control. The PID controller parameters were optimized based on genetic algorithm and harmonic search method. The simulation results show the robustness of the proposed controller.

The sliding mode control strategy was proposed for an underwater vehicle position control in Tabar et al. [12]. The controller was applied to overcome the effect of the disturbances. Zhou et al. [13] proposed a state feedback sliding mode controller for a nonlinear dynamic system of an underwater vehicle with disturbance consideration. The simulation results show a good performance.

In this paper, a model dependent controller for autonomous underwater vehicle is proposed. The controller was developed to include the nonlinear dynamics of the vehicle. Then, a model independent controller was presented, and it is results were compared with that of the former controller.

In the following, section II presents the underwater vehicle dynamical model. Section III provides the description of the designed nonlinear feedback control algorithm. Section IV provides simulation results. Our conclusion and future work are given in section $\mathrm{V}$.

\section{AUV MODELLING}

The nonlinear dynamical model of a 6DOF underwater vehicle can be described based on two reference frames; fixed reference frame (inertial reference frame) I and the body frame (motion reference frame) B, shown in Fig. 1. The dynamics and kinematics of the vehicle are expressed as follows [14]:

$$
\begin{aligned}
& M \dot{v}+C(v) v+D(v) v+g(\eta)=\tau \\
& \dot{\eta}=J(\eta) v
\end{aligned}
$$

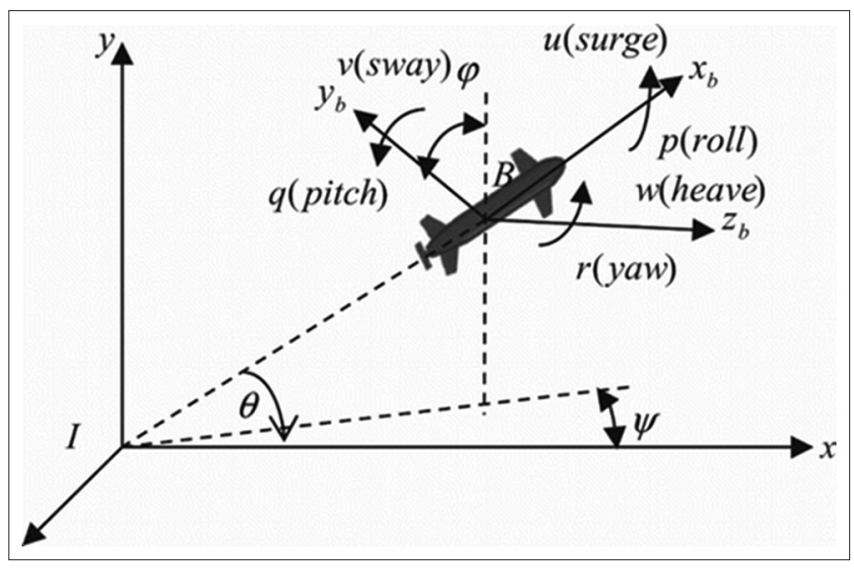

Fig. 1. Underwater vehicle frames

Where, $M=M^{\mathrm{T}}$ is a positive $\mathrm{R}^{6 \times 6}$ inertia matrix with the added masses, $C(v)=-C(v)^{\mathrm{T}}$ is $\mathrm{R}^{6 \times 6}$ Coriolis and centripetal matrix, $D(v)$ is a positive $\mathrm{R}^{6 \times 6}$ hydrodynamic damping matrix, $g(\eta)$ is $\mathrm{R}^{6 \times 1}$ gravitational and buoyancy vector, $\tau=\left[\tau_{\mathrm{X}}, \tau_{\mathrm{P}}, \tau_{\mathrm{Z}}, \tau_{\mathrm{K}}, \tau_{\mathrm{M}}, \tau_{\mathrm{N}}\right]^{\mathrm{T}}$ is $\mathrm{R}^{6 \times 1}$ forces and torque input vector, $v=[\mathrm{u}, \mathrm{v}, \mathrm{w}, \mathrm{p}, \mathrm{q}, \mathrm{r}]^{\mathrm{T}}$ is the linear and angular velocity vector, $\eta=[\mathrm{x}, \mathrm{y}, \mathrm{z}, \varphi, \theta, \psi]^{\mathrm{T}}$ is $\mathrm{R}^{6 \times 1}$ motion vector in surge, sway, heave, roll, pitch, and yaw, respectively, and $J(\eta)$ is $\mathrm{R}^{6 \times 6}$ body frame to inertial frame transformation matrix.

$$
\begin{aligned}
J(\eta) & =\left[\begin{array}{ccc}
c \psi c \theta & -s \psi c \phi+c \psi s \theta s \phi & c \psi s \theta c \phi+s \psi s \phi \\
s \psi c \theta & s \psi s \theta s \phi+c \psi s \phi & s \psi s \theta c \phi-c \psi s \phi \\
-s \theta & c \theta s \phi & c \theta s \phi \\
0 & 0 & 0 \\
0 & 0 & 0 \\
0 & 0 & 0 \\
0 & 0 & 0 \\
0 & 0 & 0 \\
0 & 0 & 0 \\
1 & s \phi t \theta & c \phi t \theta \\
0 & c \phi & -s \phi \\
0 & \frac{s \phi}{c \theta} & \frac{c \phi}{c \theta}
\end{array}\right]
\end{aligned}
$$

Where, $s, c$, and $t$ are sine, cosine, and tan.

Assuming that the vehicle is symmetry about the three planes, the vehicle is operate in low speed, roll, and pitch movement is neglected, the body frame is considered to be at same position of the center of gravity, no disturbance is considered, and all the dynamic states can be decoupled, and the dynamical system Eq. (1) can be rewritten as follows: 
$M \dot{v}+D(v) v+g(\eta)=\tau$

$\dot{\eta}=J(\eta) v$

Where,

$$
\begin{gathered}
M=\left[\begin{array}{cccc}
m+X_{\dot{u}} & 0 & 0 & 0 \\
0 & m+Y_{\dot{v}} & 0 & 0 \\
0 & 0 & m+Z_{\dot{\nu}} & 0 \\
0 & 0 & 0 & I_{x}+K_{\dot{p}} \\
0 & 0 & 0 & 0 \\
0 & 0 & 0 & 0 \\
0 & 0 \\
0 & 0 \\
0 & 0 \\
0 & 0 \\
I_{y}+M_{\dot{q}} & 0 \\
0 & I_{z}+N_{\dot{r}}
\end{array}\right]
\end{gathered}
$$

and

$$
\begin{gathered}
D=\left[\begin{array}{ccc}
X_{u}+X_{u|u|}|u| & 0 & 0 \\
0 & Y_{v}+Y_{v|v|}|v| & 0 \\
0 & 0 & Z_{w}+Z_{w|p|}|w| \\
0 & 0 & 0 \\
0 & 0 & 0 \\
0 & 0 & 0 \\
0 & 0 & 0 \\
0 & 0 & 0 \\
0 & 0 & 0 \\
K_{p|p|}|p| & 0 & 0 \\
0 & K_{q}+K_{q|q|}|q| & 0 \\
0 & 0 & N_{r}+N_{r|r|}|r|
\end{array}\right]
\end{gathered}
$$

and only four degrees of freedom are considered to control the vehicle, i.e., control $(x, y, z)$ and $\psi$ states.

\section{CONTROLLER DESIGN}

In this section, the aim is to design a feedback control algorithm for the path following problem of the underwater vehicle. To this end, the first equation of Eq. 2 will be used. Our control scheme consists of two approaches. The first approach is to design a model dependent controller to find the desired control vector $\tau$. The second approach is to design a model independent control algorithm to obtain the desired control vector $\tau$. The controllers' stability analyses are guaranteed based on Lyapunov function as exponential and asymptotic stability, respectively.

The main task is to derive the underwater vehicle toward the desired position $\eta_{d}$ from the initial position to satisfy the following equilibrium point.

$\lim _{t \rightarrow \infty} \tilde{\eta}=\lim _{t \rightarrow \infty}\left(\eta_{d}-\eta\right)=0$

Now, the following theorem can be addressed:

Theorem 1: Considering the dynamics of Eq.2 under the feedback control law of the form:

$$
\tau=\mathrm{M} \dot{\mathrm{v}}+\mathrm{D}(\mathrm{v}) \mathrm{v}+\mathrm{g}(\eta)+\dot{\tilde{\eta}}+\dot{\tilde{v}}
$$

Where, $\dot{\tilde{\eta}}=-\mathrm{k}_{\mathrm{p}} \tilde{\eta}, \dot{\tilde{\mathrm{v}}}=-\mathrm{k}_{\mathrm{d}} \tilde{\mathrm{v}}, k_{p}$ and $k_{d}$ are diagonal matrices, $\tilde{\eta}=\eta_{d}-\eta$ is the motion vector error, and is the linear and angular velocity error. Then, the closed loop system of Eq.2 and Eq.4 is exponentially stable.

Proof: Let us suggest the following Lyapunov candidate:

$$
\mathrm{V}=\frac{1}{2} \tilde{\eta}^{\mathrm{T}} \tilde{\eta}+\frac{1}{2} \tilde{\mathrm{v}}^{\mathrm{T}} \tilde{\mathrm{v}}
$$

Calculating the time derivative of the proposed Lyapunov function we obtain:

$\dot{\mathrm{V}}=\tilde{\eta}^{\mathrm{T}} \dot{\tilde{\eta}}+\tilde{\mathrm{v}}^{\mathrm{T}} \dot{\tilde{\mathrm{V}}}$

Substituting the value of $\dot{\tilde{\eta}}$ and $\dot{\tilde{v}}$ into Eq.6 we get:

$\dot{V}=-\tilde{\eta}^{T} k_{p} \tilde{\eta}-\tilde{v}^{T} k_{d} \tilde{v} \leq 0$

Then, Eq.7 is less than zero leaded $k_{p}$ and $k_{d}$ are positive definite diagonal matrices, and it can be concluded based on the Barbalat's lemma [15] that the closed loop system Eq.2 and the control law Eq.4 is globally asymptotically stable, which meets the condition of Eq.3.

Now, the model independent feedback control law, i.e., it is a PD controller without the effect of the hydrodynamic 
damping, gravitational and buoyancy forces, and the inertia terms are:

$$
\tau=-\Gamma_{1} \tilde{\eta}-\Gamma_{2} \tilde{v}
$$

Where, $\Gamma_{1}$ and $\Gamma_{2}$ are positive diagonal matrices.

\section{SIMULATIONS}

The model dependent controller Eq.4 has been applied for the four state to be controlled of the autonomous underwater vehicle, which presented in Singh and Chowdhury [16]. A vehicle MATLAB simulator was implemented with the following matrices:

$\mathrm{M}=\left[\begin{array}{cccccc}99 & 0 & 0 & 0 & 0 & 0 \\ 0 & 108.5 & 0 & 0 & 0 & 0 \\ 0 & 0 & 126 & 0 & 0 & 0 \\ 0 & 0 & 0 & 1.05 & 0 & 0 \\ 0 & 0 & 0 & 0 & 1.002 & 0 \\ 0 & 0 & 0 & 0 & 0 & 29.1\end{array}\right]$

$\mathrm{D}=\left[\begin{array}{cccccc}\mathrm{d}_{1} & 0 & 0 & 0 & 0 & 0 \\ 0 & \mathrm{~d}_{2} & 0 & 0 & 0 & 0 \\ 0 & 0 & \mathrm{~d}_{3} & 0 & 0 & 0 \\ 0 & 0 & 0 & \mathrm{~d}_{4} & 0 & 0 \\ 0 & 0 & 0 & 0 & \mathrm{~d}_{5} & 0 \\ 0 & 0 & 0 & 0 & 0 & \mathrm{~d}_{6}\end{array}\right]$

With

$\mathrm{d}_{1}=10+227.18|\mathrm{u}|$

$\mathrm{d}_{2}=405.41[\mathrm{v}]$

$\mathrm{d}_{3}=10+227.18|\mathrm{w}|$

$\mathrm{d}_{4}=0.05+5.21|\mathrm{p}|$

$\mathrm{d}_{5}=0.025+3.22|\mathrm{q}|$

$\mathrm{d}_{6}=1.603+12.937|\mathrm{r}|$

$g(\eta)=\left[\begin{array}{c}0 \\ 0 \\ -19.6 \\ 0 \\ 0 \\ 0\end{array}\right]$
To validate the proposed model dependent control performance, two paths are considered. Then, the vehicle retested under the model independent PD controller Eq. 8 and its results are compared with that of the model dependent controller Eq.4.

First, the following desired path was tested:

$$
\left\{\begin{array}{c}
\mathrm{x}_{\mathrm{d}}=20 \sin (\mathrm{t} / 10) \\
\mathrm{y}_{\mathrm{d}}=20 \cos (\mathrm{t} / 10) \\
\mathrm{z}_{\mathrm{d}}=10 \\
\psi_{\mathrm{d}}=\pi / 2
\end{array}\right.
$$

Then, the following desired path was tested:

$$
\left\{\begin{array}{c}
x_{d}=5 t \\
y_{d}=3 t \\
z_{d}=20 \\
\psi_{d}=\pi / 4
\end{array}\right.
$$

The vehicle was started from zero initial condition in both cases.

Figs. 2-5 show the typical results when the vehicle was commanded to follow the first desired path under the proposed controller compared with that of the PD controller. While Figs. 6-9 present the results obtained when the second path was used.

Figs. 2 and 6 present the motion of the vehicle toward $x$-axis under the two controllers in the first and the second

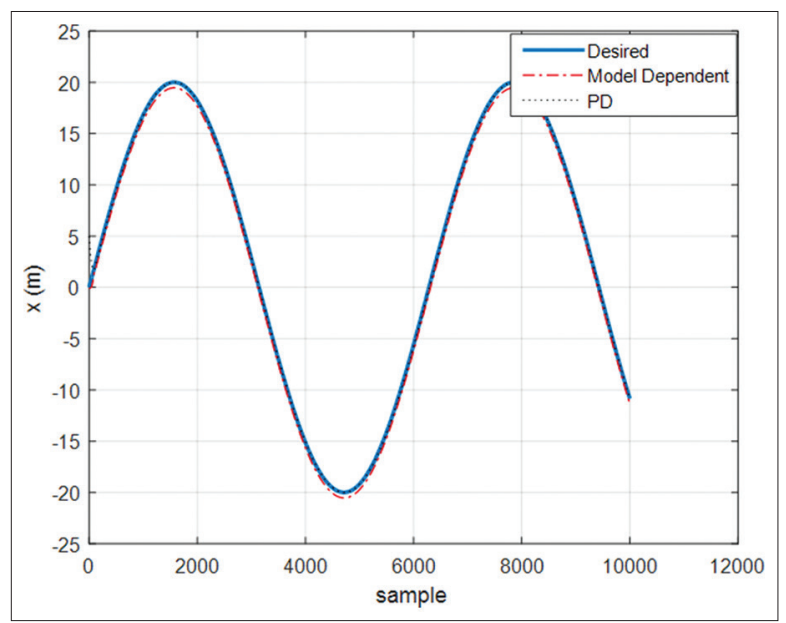

Fig. 2. Motion of the vehicle toward $x$-direction in first path 
Wesam M. Jasim: Model Dependent Controller for Underwater Vehicle

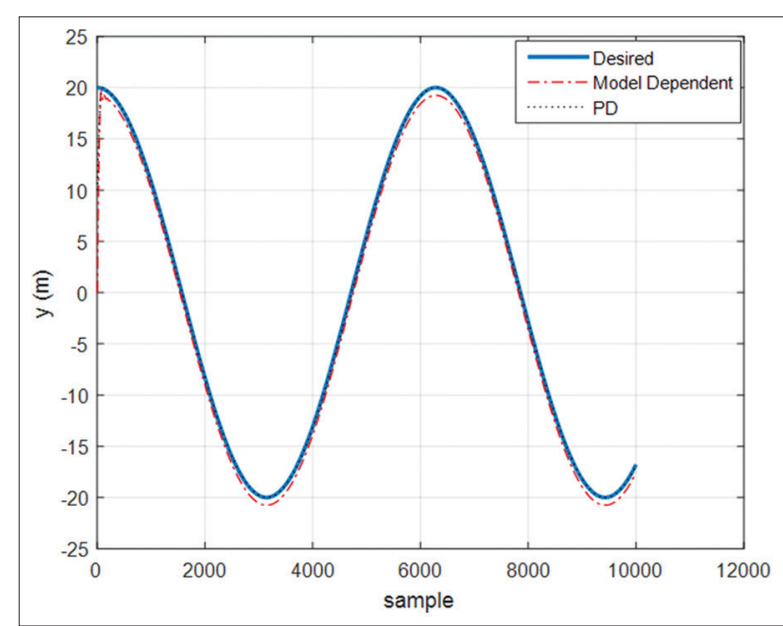

Fig. 3. Motion of the vehicle toward y-direction in first path

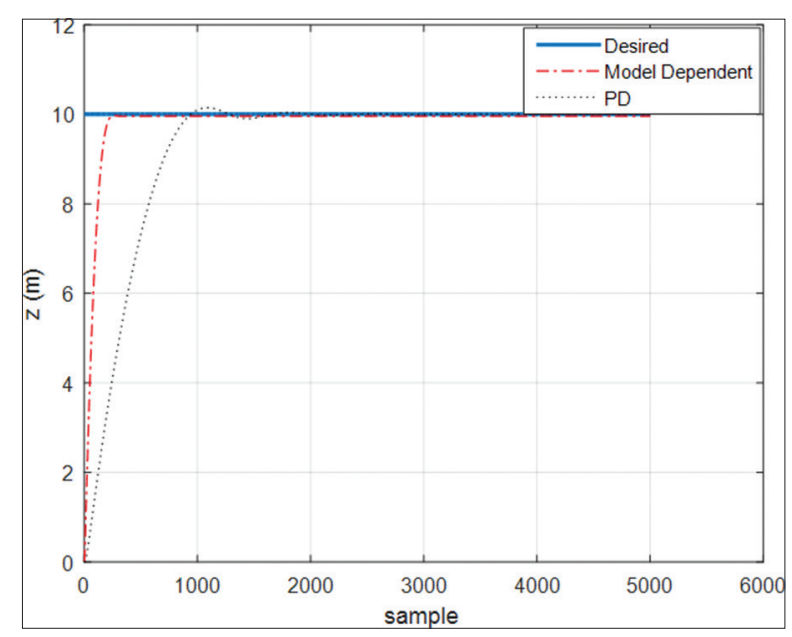

Fig. 4. Motion of the vehicle toward z-direction in first path

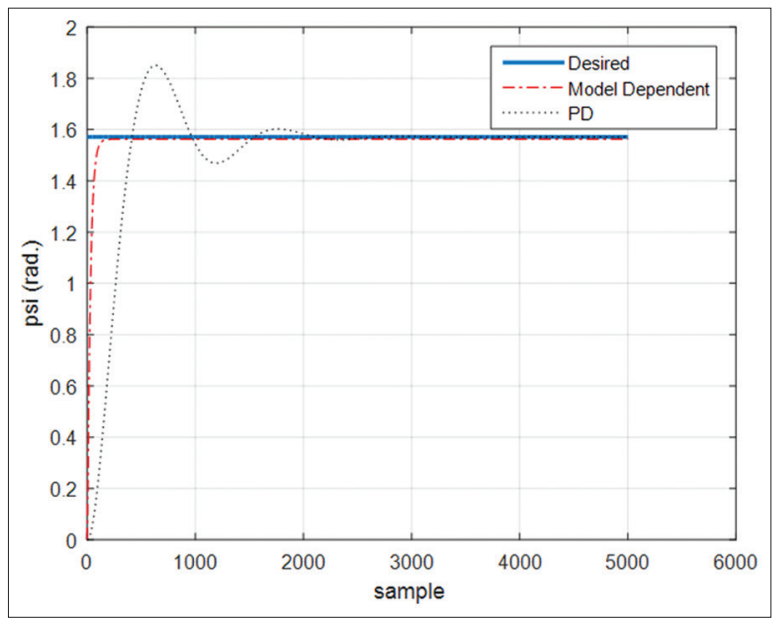

Fig. 5. Rotating of the vehicle along z-axis in first path

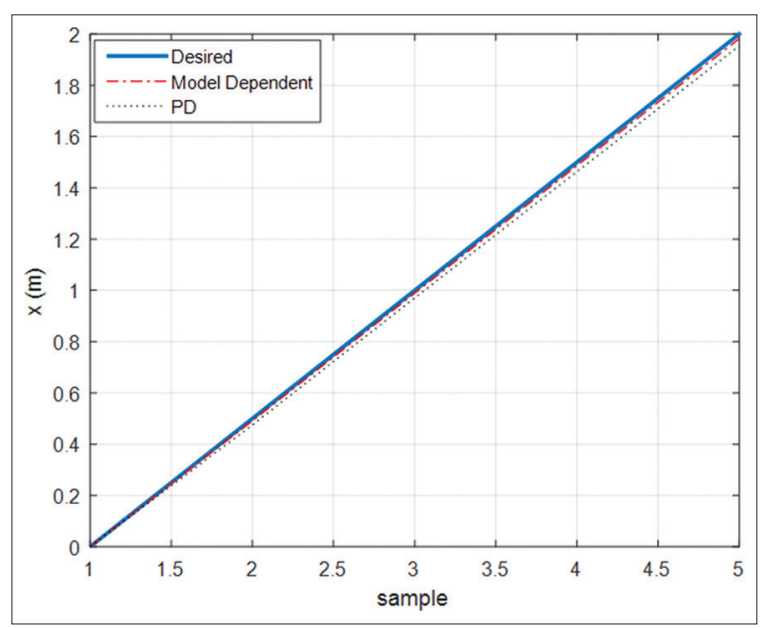

Fig. 6. Motion of the vehicle toward x-direction in second path

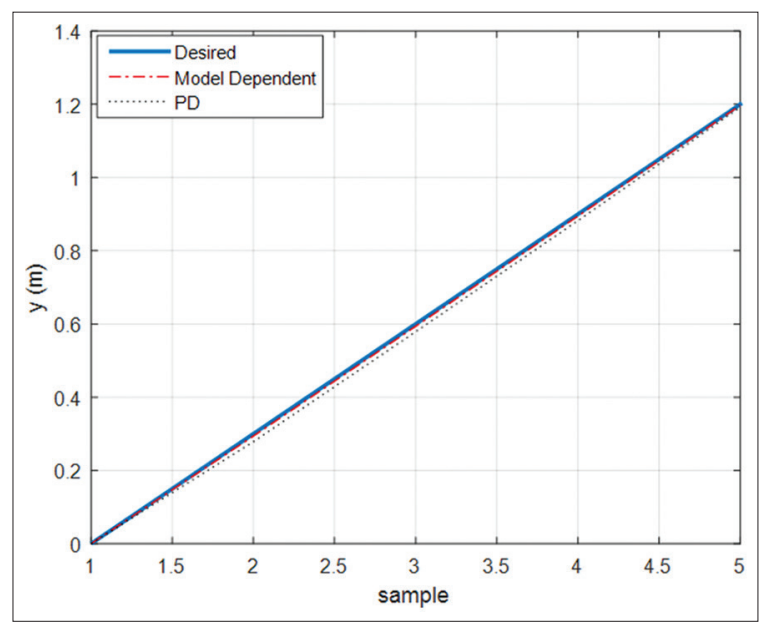

Fig. 7. Motion of the vehicle toward y-direction in second path

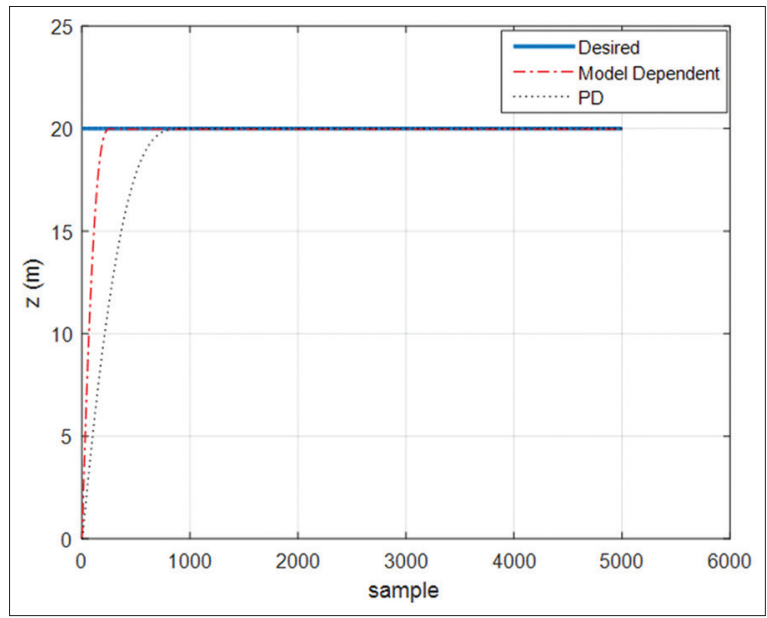

Fig. 8. Motion of the vehicle toward z-direction in second path 


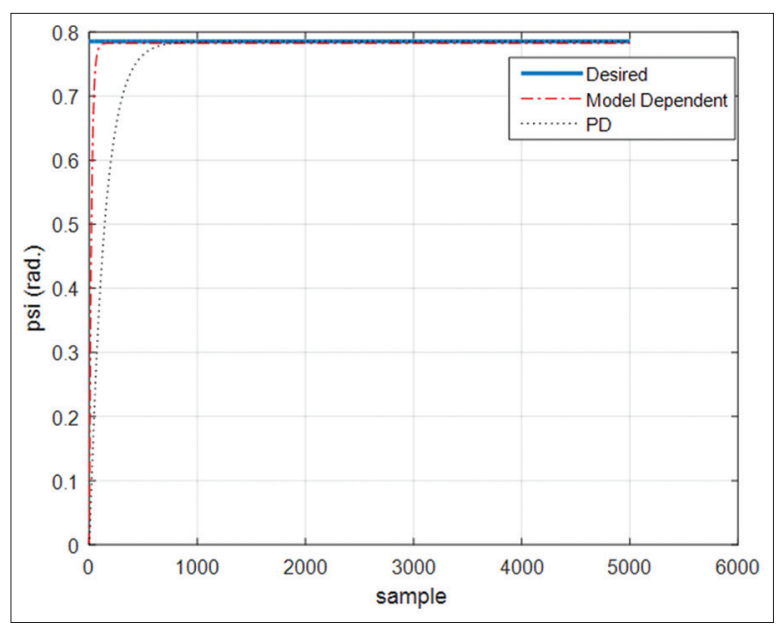

Fig. 9. Rotation of the vehicle along z-axis in second path

cases, respectively. It can be seen that the performance of the vehicle under the proposed controller is faster than that of PD controller to catch the desired path with smaller oscillation.

From Figs. 3, 4, 7, and 8, one can conclude that the vehicle moved toward $y$ and $z$-axes with very small error when the proposed controller was used compared with some over shoot when the PD controller was used.

The performance of the rotation angle $\psi$ was obtained in Figs. 5 and 9 for the first and the second cases, respectively. In these figures, no oscillation was appearing in both cases, but the proposed controller performs faster than the PD one.

It is quite obvious that the performance of the vehicle path following under the proposed controller is much better than the performance of the vehicle under the PD controller.

\section{CONCLUSIONS}

The design of a model dependent controller of an underwater vehicle has been addressed in this paper. The proposed controller includes the vehicle nonlinear dynamic terms. The control system stability was guaranteed through Lyapunov theory. The simulation results of using the proposed controller show better performance compared with that of PD controller. Our work toward this subject is to apply the proposed controller for a swarm of underwater vehicle control problem.

\section{REFERENCES}

[1] E. Roche, O. Sename and D. Simon. "LPV/H $\mathrm{H}_{\infty}$ control of an autonomous underwater vehicle AUV," Procedings of the European Control Conference, 2009.

[2] N. M. Patel, S. E. Gano and J. E. Renaud. "Simulation model of an autonomous underwater vehicle for design optimization," $45^{\text {th }}$ AIAA/ASME/ASCE/AHS/ASC Structures, Structural Dynamics and Materials Conference, pp. 1-15, Apr. 2004.

[3] B. Ferreira, M. Pinto, A. Matos and N. Cruz. "Control of the MARES autonomous underwater vehicle," in OCEANS, pp. 1-10. Oct. 2009.

[4] J. H. A. Vervoort. "Modeling and Control of an Unmanned Underwater Vehicle." master thesis, University of Technology Eindhoven, 2008.

[5] M. P. R. Prasad and A. Swarup." Position and Velocity Control of Remotely Operated Underwater Vehicle using Model Predictive Control." Indian Journal of Geo-Marine Sciences, vol. 44, no. 12, pp. 1920-1927, 2015.

[6] M. G. Joo and Z. Qu." An autonomous underwater vehicle as an underwater glider and its depth control." International Journal of Control, Automation, and Systems, vol. 13, no. 5, pp. 1212-1220, 2015.

[7] R. Mohd-Mokhtar, M. H. R. Aziz, M. R. Arshad, A. B. Husaini and M. M. Noh. "Model identification and control analysis for underwater thruster system." Indian Journal of Geo-Marine Sciences, vol. 42, no. 8, pp. 992-998, 2013.

[8] G. A. Elnashar. "Dynamics modelling, performance evaluation and stability analysis of an autonomous underwater vehicle." International Journal of Modelling, Identification and Control, vol. 21, no. 3, pp. 306-320, 2014.

[9] K. Mukheriee, I. N. Kar and R. K. P. Bhatt. "Adaptive gravity compensation and region tracking control of an AUV without velocity measurement." International Journal of Modelling, Identification and Control, vol. 25, no. 2, pp. 154-163, 2016.

[10] L. V. Steenson, S. R. Turnock, A. B. Phillips, C. Harris, M. E. Furlong, E. Rogers and L. Wang. "Model predictive control of a hybrid autonomous underwater vehicle with experimental verification," in Proc. Of the Institution of Mechanical Engineers, Part M: Journal of Engineering for the MaritimeEnvironment, 2013.

[11] A. Rathore and M. Kumer. "Robust steering control of autonomous underwater vehicle: Based on PID tuning evolutionary optimization technique." International Journal of Computer Applications, vol. 117, no. 18, pp. 1-6, 2015.

[12] A. F. Tabar, M. Azadi and A. Alesaadi. "Sliding mode control of autonomous underwater vehicles." International Journal of Computer, Electrical, Automation, Control and Information Engineering, vol. 8, no. 3, pp. 546-549, 2014.

[13] H. Y. Zhou, K. Z. Liu and X. S. Feng. "State feedback sliding mode control without chattering by constructing hurwitz matrix for AUV movement." International Journal of Automation and Computing, vol. 8, no. 2, pp. 262-268, 2011.

[14] T. I. Fossen, Guidance and Control of Ocean Vehicles. New York: John Wiley \& Sons, 1994.

[15] J. J. Slotine, and Li, W. Applied Nonlinear Control. New Jersey: Prentice Hall, 1991.

[16] M. P. Singh and B. Chowdhury. Control of Autonomous Underwater Vehicles. Rourkela: Bachelor of Technology in Electrical Engineering, National Institute of Technology, 2011. 